\title{
MerTK inhibition in tumor leukocytes decreases tumor growth and metastasis
}

\author{
Rebecca S. Cook, ${ }^{1,2}$ Kristen M. Jacobsen, ${ }^{3,4}$ Anne M. Wofford, ${ }^{5}$ Deborah DeRyckere, ${ }^{4}$ \\ Jamie Stanford, ${ }^{1}$ Anne L. Prieto, ${ }^{6}$ Elizabeth Redente, ${ }^{7}$ Melissa Sandahl, ${ }^{8}$ Debra M. Hunter, ${ }^{8}$ \\ Karen E. Strunk, ${ }^{8}$ Douglas K. Graham, ${ }^{3,4}$ and H. Shelton Earp III ${ }^{8}$

\begin{abstract}
${ }^{1}$ Department of Cancer Biology, Vanderbilt University School of Medicine, Nashville, Tennessee, USA. 2Ingram Cancer Center, Nashville, Tennessee, USA.
${ }^{3}$ Department of Integrated Immunology and ${ }^{4}$ Department of Pediatrics, University of Colorado, Anschutz Medical Campus, Aurora, Colorado, USA.

${ }^{5}$ Wake Forest School of Medicine, Winston-Salem, North Carolina, USA. ${ }^{6}$ Department of Psychological and Brain Science, Indiana University, Bloomington, Indiana, USA. ${ }^{7}$ Department of Pediatrics, National Jewish Health, Denver, Colorado, USA. ${ }^{8}$ Departments of Pharmacology and Medicine and UNC Lineberger Comprehensive Cancer Center, University of North Carolina School of Medicine, Chapel Hill, North Carolina, USA.
\end{abstract}

\begin{abstract}
MerTK, a receptor tyrosine kinase (RTK) of the TYRO3/AXL/MerTK family, is expressed in myeloid lineage cells in which it acts to suppress proinflammatory cytokines following ingestion of apoptotic material. Using syngeneic mouse models of breast cancer, melanoma, and colon cancer, we found that tumors grew slowly and were poorly metastatic in $\mathrm{MerTK}^{-/-}$mice. Transplantation of $\mathrm{MerTK}^{-/-}$bone marrow, but not wild-type bone marrow, into lethally irradiated $M M T V-P y V m T$ mice (a model of metastatic breast cancer) decreased tumor growth and altered cytokine production by tumor $\mathrm{CD}_{11} \mathrm{~b}^{+}$cells. Although MerTK expression was not required for tumor infiltration by leukocytes, $\mathrm{MerTK}^{-/-}$leukocytes exhibited lower tumor cell-induced expression of wound healing cytokines, e.g., IL-10 and growth arrest-specific 6 (GAS6), and enhanced expression of acute inflammatory cytokines, e.g., IL-12 and IL-6. Intratumoral CD8 ${ }^{+} \mathrm{T}$ lymphocyte numbers were higher and lymphocyte proliferation was increased in tumor-bearing $\mathrm{MerTK}^{-/}$mice compared with tumor-bearing wild-type mice. Antibody-mediated $\mathrm{CD8}^{+} \mathrm{T}$ lymphocyte depletion restored tumor growth in $\mathrm{MerTK}^{-/}$mice. These data demonstrate that MerTK signaling in tumor-associated $\mathrm{CD}_{11 \mathrm{~b}^{+}}$leukocytes promotes tumor growth by dampening acute inflammatory cytokines while inducing wound healing cytokines. These results suggest that inhibition of MerTK in the tumor microenvironment may have clinical benefit, stimulating antitumor immune responses or enhancing immunotherapeutic strategies.
\end{abstract}

\section{Introduction}

MerTK is a member of a receptor tyrosine kinase (RTK) family that also includes AXL and TYRO3. Family members undergo ligand-induced homodimerization, followed by catalytic tyrosine kinase activation and intracellular signaling (1-4). Cross-phosphorylation has also been demonstrated within this RTK family, suggesting heterodimerization (5). These RTKs are widely expressed in many epithelial tissues and in cells of the immune, nervous, and reproductive systems $(2,6)$. The MerTK ligands include growth arrest-specific $6(\operatorname{GAS} 6)(7,8)$, protein-S $(9,10)$, tubby and tubbylike protein-1 (TULP1) (11), and galectin-3 (12). Several of these ligands are present in serum, and all are expressed locally in some tissues. These ligands bind to the extracellular domain of MerTK, resulting in tyrosine kinase activation.

With respect to neoplastic diseases, MerTK is expressed in nonneoplastic cells found in the tumor microenvironment. MerTK is also ectopically expressed or overexpressed in many hematologic and epithelial malignant cells. Moreover, expression of MerTK and GAS6 correlates with poor prognosis or chemoresistance in some human tumor types $(1,2,13-19)$. However, the mechanisms by which increased MerTK signaling contributes to tumor malignancy remain unknown.

Conflict of interest: The authors have declared that no conflict of interest exists.

Note regarding evaluation of this manuscript: Manuscripts authored by scientists associated with Duke University, The University of North Carolina at Chapel Hill, Duke-NUS, and the Sanford-Burnham Medical Research Institute are handled not by members of the editorial board but rather by the science editors, who consult with selected external editors and reviewers.

Citation for this article: J Clin Invest. 2013;123(8):3231-3242. doi:10.1172/JCI67655
Studies using mice devoid of MerTK revealed its critical role at the interface of innate and adaptive immunity $(4,20,21)$. Innate immunity requires rapid and robust activation in response to pathogens or wounding. However, this response must be restrained to prevent inflammation-associated tissue damage or immunity against self-antigens. MerTK signaling plays a central role in dampening the innate immune response in DCs and macrophages (21). One mechanism by which MerTK performs this task is through efferocytosis, the physiological process by which apoptotic cells are engulfed by phagocytes (22). MerTK ligands, including GAS6, simultaneously bind to MerTK expressed on phagocytes and to phosphatidylserine presented on the outer plasma membrane leaflets of apoptotic cells $(23,24)$. This complex ligand (GAS6 bound to externalized phosphatidylserine) activates MerTK tyrosine kinase signaling, initiates phagocytosis of apoptotic material, and drives transcriptional changes that cause suppression of proinflammatory cytokines, such as IL-12, and increases in inflammatory repressors, such as IL-10 $(25,26)$. Therefore, MerTK-mediated efferocytosis is necessary to maintain tissue homeostasis in organs harboring abundant apoptotic materials, such as the retina and the postlactational mammary gland $(27,28)$.

MerTK similarly dampens TLR-induced production of proinflammatory cytokines, such as IL-6, IL-12, and type I interferons (IFNs), which fail to be downregulated in $\operatorname{MerTK}^{-/-}$mice (4, 24, 29). For example, low doses of lipopolysaccharide in $\mathrm{MerTK}^{-/-}$mice resulted in death from endotoxic shock associated with high levels of TNF- $\alpha$ (30). Failure to dampen acute innate immunity leads to secondary pathological activation of $\mathrm{T}$ and $\mathrm{B}$ lymphocytes directed at self-antigens $(4,26,29,31,32)$. This is especially important 
A

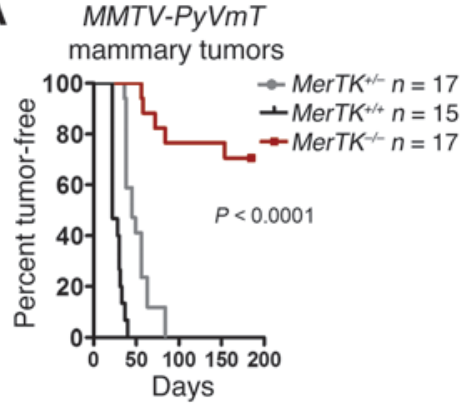

B

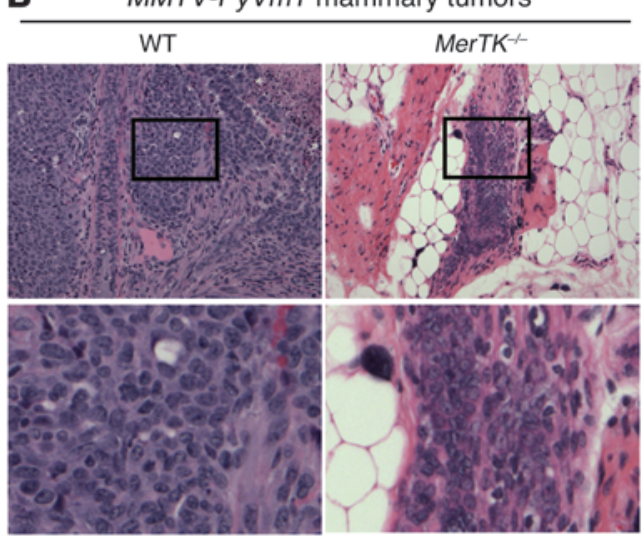

$\mathrm{B} 16: \mathrm{F} 10$

intradermal tumors

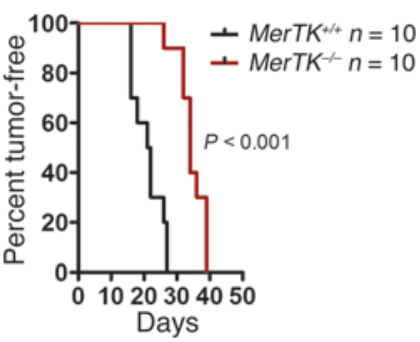

C

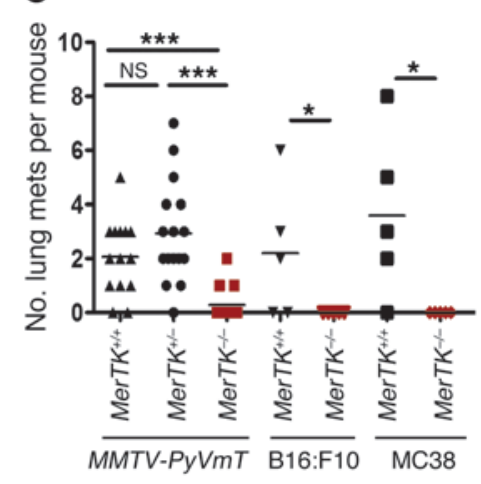

Figure 1

Decreased tumor malignancy in MerTK ${ }^{-/-}$microenvironment. (A) MMTV-PyVmT mammary tumor cells injected into the mammary fat pads and B16:F10 tumor cells injected intradermally formed tumors with a delayed latency in $\mathrm{MerTK}^{-/-}$mice as compared with that in $\mathrm{MerTK}^{+/+}$and $\mathrm{MerTK}^{+/-}$ mice. $P$ values comparing $\mathrm{MerTK}^{+/+}$to $\mathrm{MerTK}^{-/}$were determined using the log-rank (Mantel-Cox) test. (B) H\&E-stained sections of tumors harvested 30 days after tumor cell injection in MerTK+/+ mice and 180 days after tumor cell injection in $\mathrm{MerTK}^{-/-}$mice. Boxed regions are shown at higher magnification below and indicate the monotonous sheets of tumor cells in $\mathrm{MerTK}^{+/+}$mice and the compartmentalized nature of the implanted tumor cells in $\mathrm{MerTK}^{-1-}$ mice. Original magnification, $\times 100$ (top row); $\times 600$ (bottom row). (C) Lung metastases were enumerated in tumor-bearing mice at 14 days after MMTV-PyVmT tumor palpation $(n=14-16)$, at 7 days after B16:F10 tumor palpation $(n=5)$, and at 7 days after MC38 tumor palpation of subcutaneously implanted MC38 cells. Horizontal bars represent average numbers of lung metastases per mouse. $P$ values were calculated using Student's $t$ test for MMTV-PyVmT tumors (comparing MerTK $\mathrm{K}^{++}$to $\mathrm{MerTK}^{-I^{-}}$) and using a 1-tailed Mann-Whitney test for B16:F10 and MC38 tumors. ${ }^{*} P<0.05 .{ }^{* \star} P<0.001$.

given that apoptotic cells accumulate in the absence of MerTK $(23,28)$, providing an enriched source for intracellular "self" antigens in the context of heightened acute inflammatory signals and enhanced B and T lymphocyte activity. Therefore, MerTK signaling on macrophages and DCs lies at the interface of the innate and adaptive immune systems. Combined loss of MerTK, AXL, and TYRO3 results in a highly active autoimmune state, with massive lymphocyte proliferation and lupus-like autoimmunity (33).

Numerous studies indicate that tumor-associated macrophages correlate with a poor prognosis in patients with cancer (34-36). Reduction of macrophage presence in mouse models of breast cancer by genetic loss of CSF1, or by antibody-mediated blockade of CSF1 receptor signaling, substantially reduced tumor metastasis $(37,38)$, demonstrating that malignant tumor progression is promoted by macrophages. Tumor-associated macrophages generally secrete wound healing cytokines that increase growth of epithelial tumor and stromal cells in experimental models (39-41).
Tumor-associated macrophages also produce immune-modulating cytokines such as IL-10, which may decrease antitumor immunity by suppressing proinflammatory cytokine expression, limiting antigen presentation, and dampening of $\mathrm{T}$ lymphocyte-mediated tumor cell cytotoxicity $(41,42)$. For example, antigen presentation in the context of increased IL-12 results in clonal expansion of $\mathrm{CD}^{+} \mathrm{T}$ lymphocytes (43); the suppression of IL-12 in the tumor microenvironment suggests a mechanism by which tumor-associated macrophage-mediated changes in antigen presentation and cytokine production may directly decrease $\mathrm{T}$ cell-mediated antitumor immunity.

Interestingly, the characteristically low IL-12/high IL-10 production observed in tumor-associated macrophages is also seen in macrophages and DCs following efferocytosis. Because MerTK appears to be a central regulator of the transition from proinflammatory to wound healing cytokine production following efferocytosis, and because MerTK-deficient macrophages produce increased proinflammatory cytokines, we tested the hypothesis that MerTK in the tumor microenvironment aids malignant tumor progression by suppressing antitumor immunity. We show here that loss of MerTK in the tumor microenvironment of $\mathrm{MerTK}^{-/-}$mice (30) slowed the establishment, growth, and metastasis of mammary tumors and melanomas in immune competent, syngeneic mice. These findings were recapitulated in spontaneous mammary tumors in recipients of $\mathrm{MerTK}^{-/-}$bone marrow transplants. Very early immune responses to syngeneic tumor cell implantation in $\mathrm{MerTK}^{-/}$mice included decreased IL-10 and increased IL-12 production, increased leukocyte proliferation, and a higher level of tumor $\mathrm{CD}^{+} \mathrm{T}$ lymphocytes as compared with early tumor-induced responses in $\mathrm{MerTK}^{++}$mice. Isolation of $\mathrm{CD} 11 \mathrm{~b}^{+}$cells from tumors revealed MerTK-dependent repression of proinflammatory cytokines. Antibody-based depletion of $\mathrm{CD}^{+} \mathrm{T}$ lymphocytes restored tumor growth in MerTK ${ }^{-1-}$ hosts. These results suggest that MerTK in the immune compartment of the tumor microenvironment suppresses innate immunity and promotes tumor progression. They also suggest that inhibition of MerTK signaling may produce an immunomodulatory, therapeutic benefit in some human tumors.

\section{Results}

Prolonged tumor latency and decreased metastasis in MerTK ${ }^{-1-}$ hosts. To determine whether host-derived MerTK in the tumor microenvironment affects tumor formation and growth, primary mouse mammary tumor cells derived from female $M M T V-P y V m T$ mice (43) (inbred into the C57BL/6 genetic background) were injected 
A

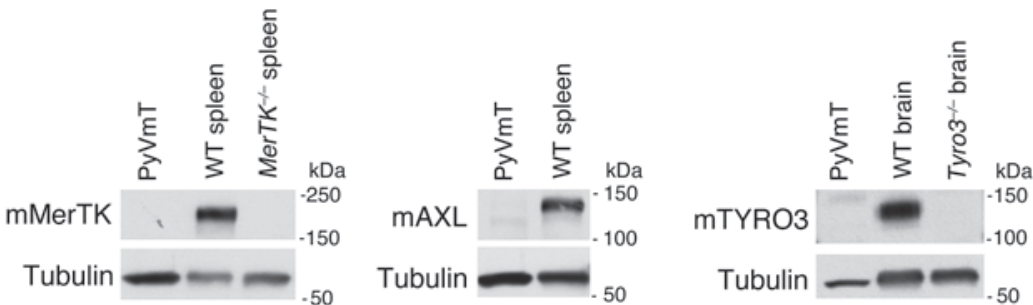

B
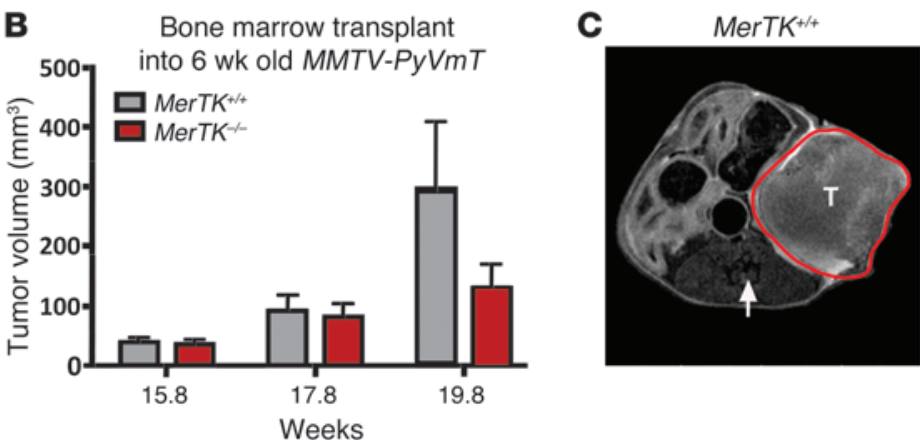

D

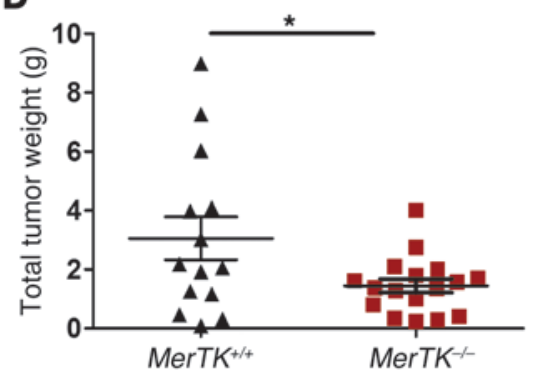

$\mathbf{E}$
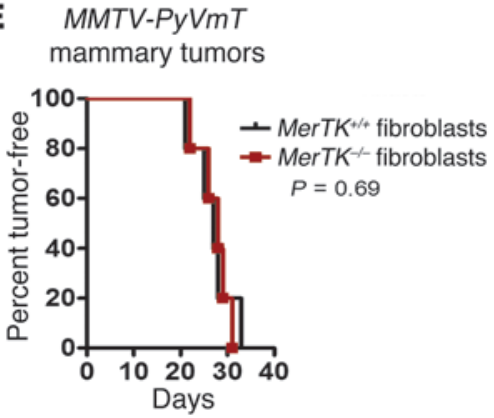

Figure 2

MerTK-deficient leukocytes confer tumor resistance to MerTK $K^{+/+}$mice. (A) Whole cell lysates harvested from MMTV-PyVmT primary mammary tumor cells were assessed by Western analysis using antibodies indicated. Whole spleen lysates harvested from $\mathrm{MerTK}^{+/+}$and $\mathrm{MerTK}^{-/-}$mice were used as positive and negative controls, respectively, for MerTK expression. Whole spleen lysates harvested from MerTK ${ }^{+/+}$ mice were used as a positive control for AXL expression. Whole brain lysates harvested from Tyro3 $3^{+/+}$and Tyro3 $^{-/-}$mice were used as positive and negative controls from TYRO3 expression. (B-D) Bone marrow harvested from $\mathrm{MerTK}^{+/+}$or $\mathrm{MerTK}^{-/-}$donors was delivered by tail vein injection into lethally irradiated 6-week-old female MMTV-PyVmT recipients. (B) Average tumor volume \pm SEM measured in live mice by MRI at 15.8, 17.8, and 19.8 weeks of age. (C) Representative transverse MRI slices of age-matched MMTV-PyVmT recipients of $\mathrm{MerTK}^{+/+}$or $\mathrm{MerTK}^{-/-}$bone marrow in the lower abdomen/pelvic region. The arrows indicate the location of the spine, while the tumor $(\mathrm{T})$ margins are identified by the red dotted line. (D) Total tumor weight measured at 21 weeks of age (time of necropsy). Horizontal bars represent average total tumor weight \pm SEM $(n=14)$. The $P$ value was calculated using Student's $t$ test. ${ }^{*} P<0.05$. (E) Mammary fibroblasts harvested from $\mathrm{MerTK}^{+/+}$and $\mathrm{MerTK}^{-/-}$mice were cotransplanted with MMTV-PyVmT tumor cells into the mammary fat pads of $\mathrm{MerTK}^{+/+}$mice, and tumor latency was measured.

orthotopically into the inguinal mammary fat pads of female wild-type $\left(\right.$ MerTK $\left.^{+/+}\right)$and MerTK $^{-/-}$recipient mice. While tumors formed in $\mathrm{MerTK}^{+/+}$mice with an average latency of 28 days, latency was delayed in $\mathrm{MerTK}^{-1-}$ littermates $(P<0.001)$. Tumors remained unpalpable in $70 \%$ of $\mathrm{MerTK}^{-/-}$mice throughout the entire study period of 198 days (Figure 1A). Mice carrying one functional MerTK allele $\left(\mathrm{MerTK}^{+/-}\right.$mice) formed tumors with an average latency of 49 days, which was intermediate between $\operatorname{MerTK}^{+/+}$mice $(P<0.001)$ and MerTK $^{-/-}$mice $(P<0.001$, Mantel-Cox test). This suggests that MerTK signaling in the stromal environment enhances tumor

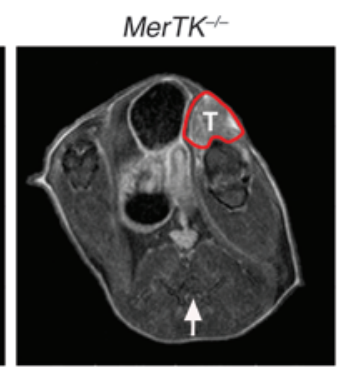

growth in a dose-dependent manner. C57BL/6-derived B16:F10 mouse melanoma cells injected orthotopically formed intradermal tumors in $\mathrm{MerTK}^{+/}$mice with an average latency of 26 days, compared with 48 days in MerTK ${ }^{-1-}$ littermates $(P<0.0001$; Figure 1A). Similarly, a third syngeneic model demonstrated prolonged latency. C57B1/6 MC38 colon cancer cells were implanted subcutaneously; in $\mathrm{MerTK}^{+/+}$mice tumors formed with an average latency of 27 days as compared with 46 days in $\mathrm{MerTK}^{-/-}$mice $(P<0.0001$, data not shown).

Histological analysis of MMTVPyVmT tumors that formed in $\mathrm{MerTK}^{-/-}$mice revealed distinct differences from those growing in $\mathrm{MerTK}^{+/}$mice. Tumors harvested from $\mathrm{MerTK}^{+/+}$mice displayed densely packed solid sheets of cells, with prominent hyperchromatic nuclei and little cytoplasm, regions of central necrosis, and invasion into skeletal muscle (Figure 1B). In contrast, tumors harvested from $\mathrm{MerTK}^{-/-}$mice were small and harbored more connective tissue as opposed to the frank cellularity of tumors in $\mathrm{MerTK}^{+/+}$ hosts (Figure 1B). MerTK $\mathrm{TH}^{-/}$mice were less prone to formation of lung metastases (Figure 1C), with only 3 out of $15 \mathrm{MerTK}^{-/-}$mice presenting with lung micrometastases derived from MMTV-PyVmT primary tumors, as compared with 13 out of 15 wild-type mice and 16 out of $17 \mathrm{MerTK}^{+/-}$mice exhibiting MMTV-PyVmT lung metastases. Lung metastases were not detected in any $\mathrm{MerTK}^{-/}$mice bearing B16:F10 tumors, while 3 out of 5 wild-type mice displayed B16:F10 lung metastases. Likewise, 4 out of 5 subcutaneously implanted MC38 colon cancer cell tumors in $\mathrm{MerTK}^{+/+}$mice yielded lung metastases, whereas none were detected in the $\mathrm{MerTK}^{-1-}$ mice (Figure 1C). These data suggest that MerTK signaling in the tumor microenvironment leads to increased tumor growth and malignant progression.

MerTK ${ }^{-/-}$lenkocytes confer tumor resistance in $\mathrm{MerTK}^{+/+}$mice. There are complex autocrine and paracrine interactions between tumor-associated macrophages and the epithelial cells in preclinical breast cancer models and presumably in human breast cancer. These have been well characterized in the polyoma middle $\mathrm{T}$ model. To rule out a potential role of MerTK in our C57/B16 
A MMTV-PyVmT mammary tumor cells 4 days after injection

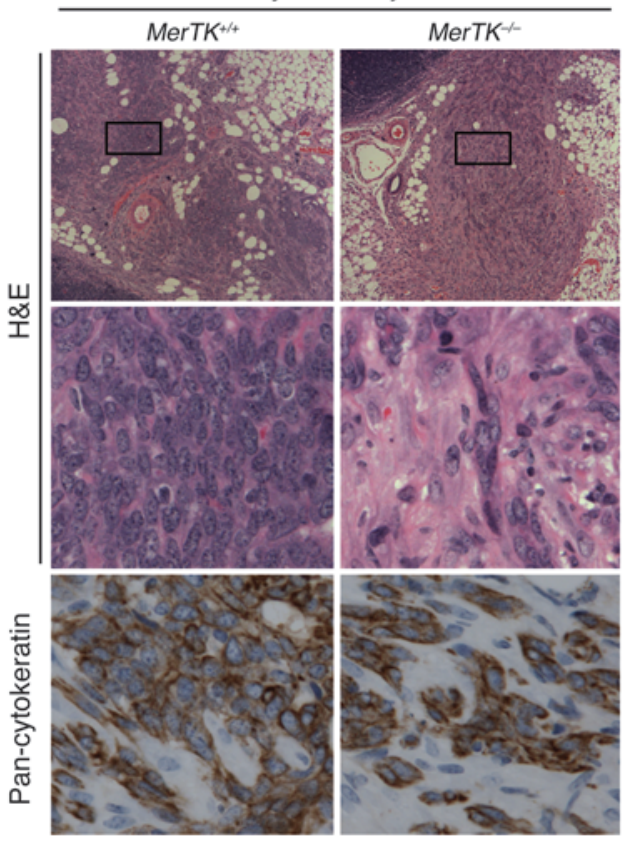

B
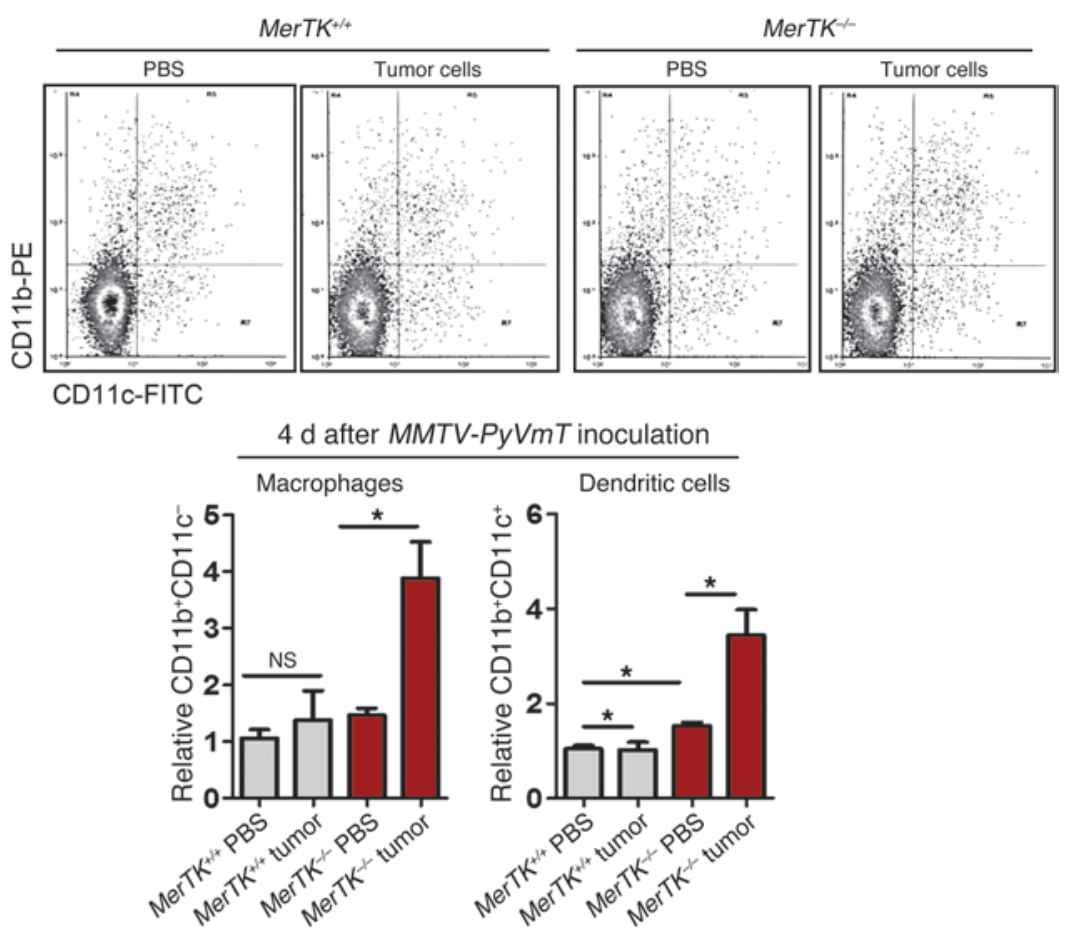

D

4 days after inoculation

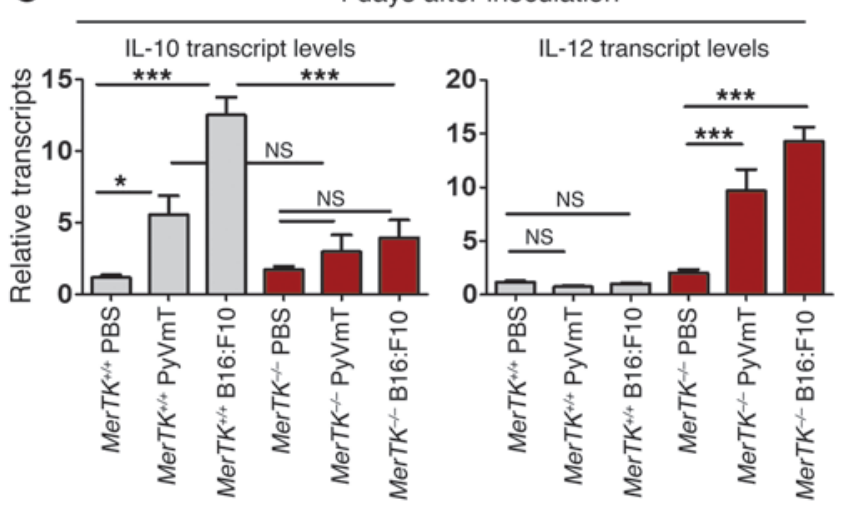

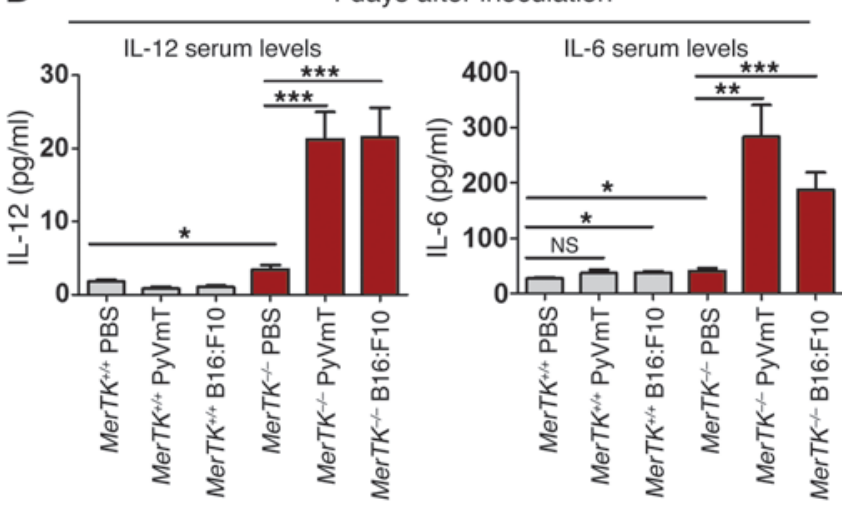

E Tumor-induced lymphocyte proliferation (BrdU) 4 days after B16:F10 IP inoculation

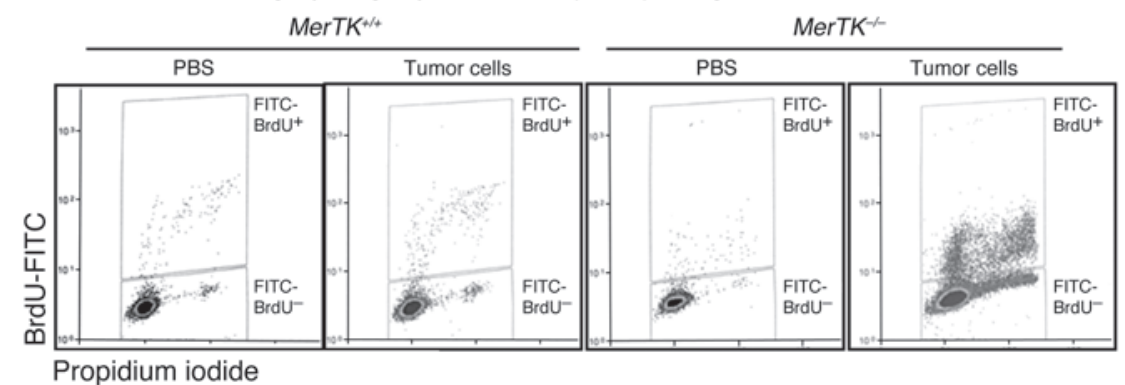




\section{Figure 3}

Altered early tumor response in MerTK-deficient mice. (A) Representative H\&E-stained and cytokeratin-stained sections of mammary glands 4 days after injection with MMTV-PyVmT cells are shown. Boxed regions are shown at higher magnification below. Original magnification, $\times 100$ (top row); $\times 600$ (bottom rows). (B) Single cell mammary suspensions harvested 4 days after intramammary tumor cell injections were stained for CD11b and CD11c. Proportions of CD11b+CD11 $\mathrm{c}^{-}$ macrophages and $C D 11 b+C D 11 c^{+}$dendritic cells relative to total PBStreated $\mathrm{MerTK}^{+/+} \mathrm{CD} 11 \mathrm{~b}+\mathrm{CD} 11 \mathrm{c}^{+}$population is shown. ${ }^{*} P<0.05$. (C) RNA harvested 4 days after PBS, MMTV-PyVmT, or B16:F10 injection into the inguinal mammary fat pad was assessed by qPCR to detect IL-10 and IL-12p40 transcripts. (D) Serum collected 4 days after injection of PBS, MMTV-PyVmT, or B16:F10 tumor cells into the inguinal mammary fat pad was assessed by ELISA to measure IL-12p70 and IL-6. (C and D) Values shown, calculated using the (C) $\delta \delta C T$ method or (D) serum levels, represent the average $\pm \mathrm{SD}(n=6)$, relative to the level detected in PBS-treated MerTK ${ }^{+/+}$samples. ${ }^{*} P<0.05$; ${ }^{* \star} P<0.01$; ${ }^{* * \star} P<0.001$. (E) Mice were inoculated with B16:F10 cells by i.p. injection. After 4 days, mice were treated with BrdU. Splenocytes harvested after 1 hour treatment with BrdU were stained with anti-BrdU and propidium iodide and assessed by flow cytometry.

PyVmT cells, we measured MerTK protein expression by Western analysis. Protein lysates from cultured tumor cells did not express MerTK. MerTK was detected in whole spleen lysates harvested from $\mathrm{MerTK}^{+/+}$mice but not $\mathrm{MerTK}^{-/-}$mice (Figure 2A). Certain paracrine ligands (e.g., GAS6) might bind to other members of this RTK family in PyVmT cells; therefore, we examined the protein expression of AXL and TYRO3. Neither AXL nor TYRO3 were expressed in the PyVmT breast cancer cells. qPCR of RNA levels confirmed these findings (data not shown).

However, as MerTK is expressed in hematopoietic cells, we used bone marrow reconstitution assays to determine the impact of MerTK ablation within bone marrow-derived subpopulations of the tumor microenvironment. We harvested bone marrow from MerTK $^{-/-} \times$UBC-GFP and MerTK ${ }^{+/+} \times U B C-G F P$ donors and delivered marrow to lethally irradiated 6 -week-old C57BL/6 female transgenic MMTV-PyVmT mice. After 4 weeks, mice exhibiting $>75 \% \mathrm{GFP}^{+}$hematopoietic cells were used for further analysis. At this time point (10 weeks of age), the majority of mice had already developed tumors, consistent with the published average tumor latency in this aggressive tumor model (44). Therefore, it was not feasible to detect potential differences in tumor latency. Instead, tumor growth was measured by MRI over the following 11 weeks, revealing reduced tumor volume in $\mathrm{MerTK}^{-/-}$bone marrow recipients as compared with that in $\mathrm{MerTK}^{+/+}$bone marrow recipients (Figure 2, B and C). Similarly, upon sacrifice at the study end point (21 weeks of age), total tumor weight was decreased nearly 2 fold in $\mathrm{MerTK}^{-/-}$bone marrow recipients as compared with that in $\mathrm{MerTK}^{+/+}$bone marrow recipients (Figure 2D). To measure the burden of apoptotic material in these tumors, immunofluorescent detection of apoptotic cells by TUNEL analysis in these late-stage tumors (19.8 weeks of age) was performed. The number of apoptotic tumor cells within $\mathrm{F} 4 / 80^{+}$macrophages was not altered in recipients of $\mathrm{MerTK}^{-/-}$bone marrow as compared to what was seen in tumors harvested from $\mathrm{MerTK}^{+/+}$bone marrow recipients (Supplemental Figure 1; supplemental material available online with this article; doi:10.1172/JCI67655DS1).

Because bone marrow-derived mesenchymal stem cells might give rise to fibroblasts in the tumor microenvironment that could modulate tumor growth and metastasis (45), we investigated the impact of $\mathrm{MerTK}^{-/-}$fibroblasts in the tumor microenvironment. $10^{6}$ mammary fibroblasts harvested from $\mathrm{MerTK}^{-/-}$and $\mathrm{MerTK}^{+/+}$ donors were cotransplanted with MMTV-PyVmT tumor cells into $\mathrm{MerTK}^{+/+}$mammary fat pads. The rate of tumor formation was similar in mice receiving $\mathrm{MerTK}^{-/-}$or $\mathrm{MerTK}^{+/+}$fibroblasts (Figure 2E). Together, these data suggest that loss of MerTK from the immune compartment, but not the fibroblast compartment, of the tumor microenvironment is sufficient to delay tumor formation.

Altered early tumor response in $\mathrm{MerTK}^{-1-}$ mice. To examine early immune response in the tumor microenvironment, inguinal mammary glands were harvested 4 days after $M M T V-P y V m T$ tumor inoculation. At this time, tumor cells were evident in the injected mammary glands of both $\mathrm{MerTK}^{+/+}$and $\mathrm{MerTK}^{-/-}$mice, as shown in $\mathrm{H} \& \mathrm{E}$-stained sections and by immunohistochemical detection of pan-cytokeratin (Figure 3A). However, higher-power magnification of the injection site revealed histological differences at this early stage, including an abundance of matrix deposition and reduced tumor cellularity in $\mathrm{MerTK}^{-/-}$samples as compared with that in $\mathrm{MerTK}^{+/+}$samples (Figure 3A). After removal of the intramammary lymph node, mammary cell suspensions were stained with antibodies against CD 11 b and CD11c to identify tumorrecruited macrophages and DCs. To control for injection-induced changes in the cell populations, we injected sterile phosphobuffered saline (PBS) into the contralateral mammary fat pads. The results revealed that, in early-stage implanted tumors, there are differences in basal macrophage and DC levels in $\mathrm{MerTK}^{-1-}$ mammary glands as compared with those in $\mathrm{MerTK}^{+/+}$mammary glands (Figure 3B). At 4 days after tumor inoculation into the mammary gland, the percentages of macrophages and DCs were relatively unchanged in $\mathrm{MerTK}^{+/+}$mice as compared to those in PBS-injected $\mathrm{MerTK}^{+/+}$mice. In contrast, tumor cell injection in $\mathrm{MerTK}^{-/-}$fat pads generated a $>4$-fold increase in macrophages and $>3.5$-fold increase in DCs over PBS-injected $\mathrm{MerTK}^{-/-}$mammary glands.

RNA isolated from single cell mammary suspensions harvested at 4 days after tumor (or PBS) inoculation revealed increased transcript levels encoding the wound healing/tolerogenic cytokine IL-10 in $\mathrm{MerTK}^{+/+}$samples but not in $\mathrm{MerTK}^{-/-}$samples (Figure 3C). Conversely, transcripts encoding the proinflammatory cytokine IL-12 were elevated 10 fold at 4 days after MMTV-PyVmT tumor cell inoculation in $\mathrm{MerTK}^{-/-}$mice but not in $\mathrm{MerTK}^{+/+}$mice. We similarly assessed $I l 10$ and Il12 mRNA levels 4 days following implantation of B16:F10 mouse melanoma cells into the mouse mammary fat pad. Tumor-recruited cells were collected 4 days after tumor cell injection. Il10 transcript levels were substantially increased in samples from tumor cell-inoculated $\mathrm{MerTK}^{+/+}$mice as compared with those from PBS-inoculated $\mathrm{MerTK}^{+/+}$mice. However, Il10 transcript levels were unchanged in cells harvested from tumor cell-inoculated $\mathrm{MerTK}^{-/-}$mice (Figure 3C). While Il12 transcript levels remained relatively unchanged in $\mathrm{MerTK}^{+/+}$cells harvested from PBS- and tumor cell-treated mice, cells harvested from $\mathrm{MerTK}^{-/-}$mice expressed more Il12 transcripts 4 days after tumor cell injection as compared with 4 days after PBS injection (Figure 3C). Serum IL-12p70 and IL-6 levels were increased 4 days after intramammary MMTV-PyVmT tumor cell injection into $\mathrm{MerTK}^{-/-}$mice, but not $\mathrm{MerTK}^{+/+}$mice, over what was seen in PBS-injected $\mathrm{MerTK}^{-/-}$and $\mathrm{MerTK}^{+/+}$mice, respectively (Figure 3D). Mammary fat pad injection of B16:F10 tumor cells also generated increased serum IL-12p70 and IL-6 levels in $\mathrm{MerTK}^{-/-}$ mice (Figure 3D). We repeated this analysis 4 days after injection of B16:F10 tumor cells i.p. into $\mathrm{MerTK}^{+/+}$and $\mathrm{MerTK}^{-/-}$mice, yielding 
A

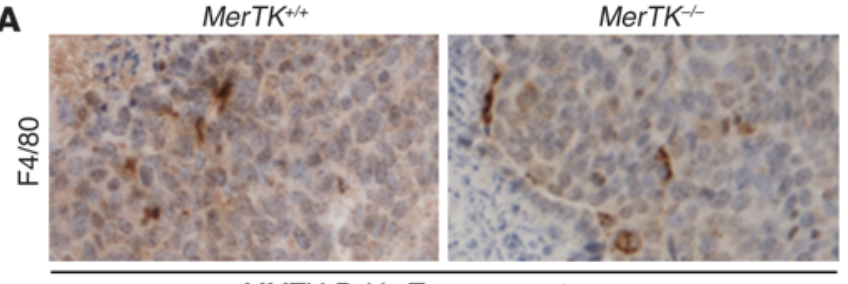

MMTV-PyVmT mammary tumors

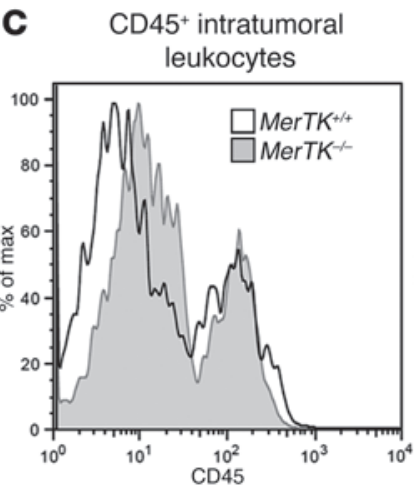

D

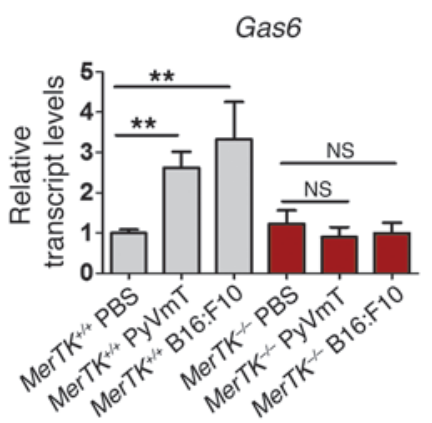

$\mathbf{F}$

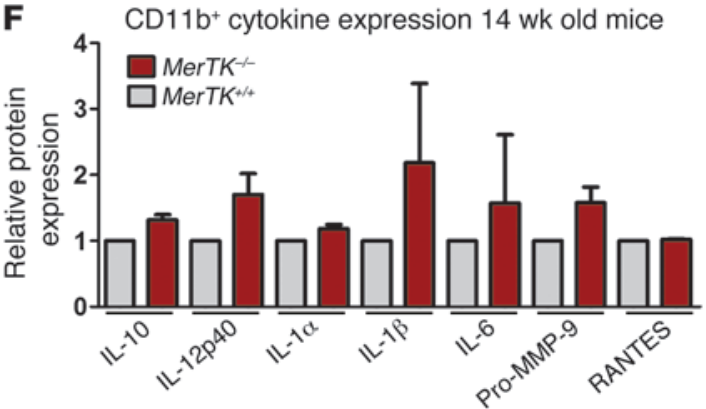

$\mathrm{CD} 45^{+} \mathrm{F} 4 / 80^{+}$intratumoral macrophages

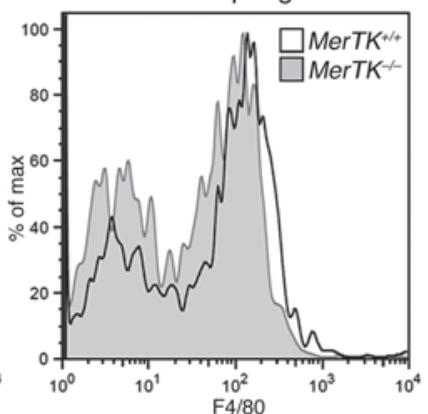

Mfge8

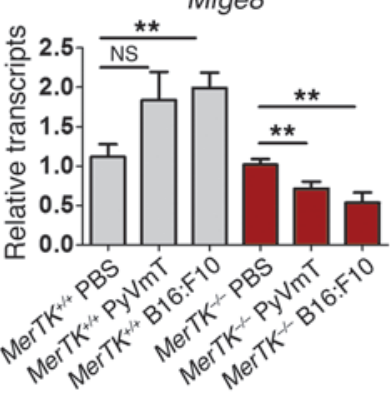

B
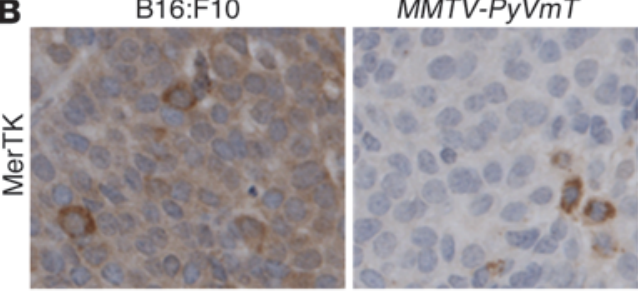

MerTK $^{+/+}$mice

Total leukocyte tumor infiltration

Macrophage
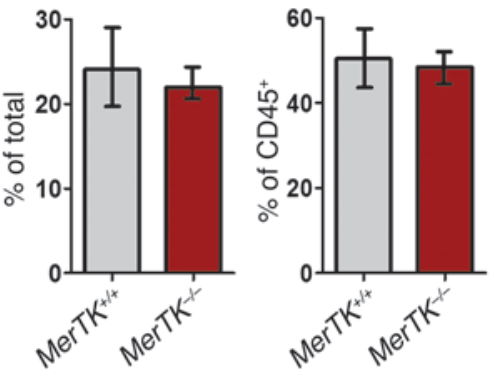

$3-4$ wk after
MMTV-PyVmT inoculation

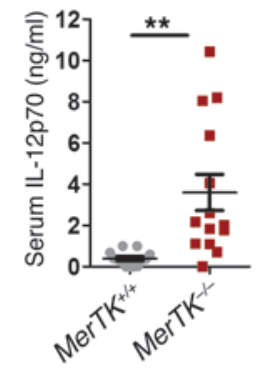

IL-6

G

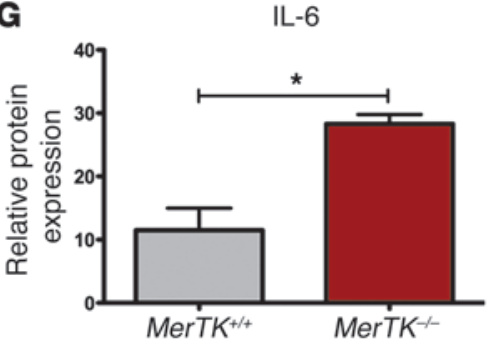

Figure 4

Altered cytokine production in MerTK ${ }^{-/-}$tumor microenvironment. (A and B) Immunohistochemistry for (A) F4/80 and (B) MerTK in tumors from (A) $\mathrm{MerTK}^{+/+}$and $\mathrm{MerTK}^{-/-}$mice and (B) MerTK ${ }^{+/+}$mice. Original magnification, $\times 400(\mathbf{A}) ; \times 600$ (B). (C) Single cell MMTV-PyVmT tumor suspensions harvested from MerTK ${ }^{+/+}$(solid line) and $\mathrm{MerTK}^{-/-}$(tinted area) mice were stained for CD45 and F4/80. Representative plots for live cells are shown. Average \pm SEM; $n=4$. (D) Total RNA harvested 4 days after PBS, MMTV-PyVmT, or B16:F10 injection was assessed by qPCR for Gas6 and Mfge8. $\delta \delta C T$ values shown represent average \pm SD $(n=6)$ relative to PBS-injected MerTK ${ }^{+/+}$values. ${ }^{* \star} P<0.01$. $(E)$ Serum IL-12p70 was measured by ELISA in serum harvested 3-4 weeks after tumor cell inoculation $(n=13)$. ${ }^{* *} P<0.01$. (F and $\left.\mathbf{G}\right)$ CD11 $\mathrm{b}^{+}$cells were harvested from MMTV-PyVmT tumors (F) 8 weeks and (G) 15 weeks after bone marrow transplant. GFP ${ }^{+}$CD $45^{+}$CD $11 b^{+}$cells were purified from tumors and cell lysates were analyzed by cytokine array. ${ }^{*} P<0.05$. 
similar results. Cells harvested by peritoneal lavage were used to assess expression of transcripts encoding IL-10 and IL-12. Il10 transcripts were elevated in cells isolated from $\mathrm{MerTK}^{+/+}$mice inoculated with B16:F10 cells by i.p. injection as compared with those in cells from $\mathrm{MerTK}^{+/+}$mice inoculated with PBS (Supplemental Figure 2). In contrast, Il10 transcripts were not elevated in $\mathrm{MerTK}^{-/}$mice inoculated with B16:F10 cells. Elevated Il12 transcripts were observed in peritoneal lavage cells harvested from tumor-inoculated $\mathrm{MerTK}^{-/-}$ mice as compared with what was seen in PBS-inoculated mice or tumor-inoculated $\mathrm{MerTK}^{+/+}$mice. Serum IL-6 was also elevated in $\mathrm{MerTK}^{-/-}$mice inoculated i.p. with B16:F10 tumor cells (Supplemental Figure 2). We used the B16:F10 i.p. inoculation model to assess splenocyte proliferative activity. BrdU labeling of mice for 1 hour, 4 days after i.p. tumor cell or PBS inoculation, revealed that BrdU incorporation into splenocytes was similar in $\mathrm{MerTK}^{++}$and MerTK ${ }^{-1}$ mice inoculated with sterile PBS and that BrdU incorporation increased in both $\mathrm{MerTK}^{+/+}$and $\mathrm{MerTK}^{-/-}$splenocytes harvested 4 days after i.p. tumor cell injection as compared with splenocytes from PBS-injected, genotype-matched mice (Figure 3E). However, the tumor-induced increase in BrdU-positive splenocytes was substantially greater in $\mathrm{MerTK} \mathrm{K}^{-/-}$mice compared with that in tumortreated $\mathrm{MerTK}^{+/+}$mice, suggesting that tumor-induced leukocyte proliferation was exaggerated in the absence of MerTK.

Altered tumor microenvironment in established tumors in $\mathrm{MerTK}^{+/+}$and $\mathrm{MerTK}^{-/-}$mice. Immunohistochemical detection of F4/80, a macrophage marker, demonstrated macrophage infiltration of orthotopic MMTV-PyVmT tumors grown in $\mathrm{MerTK}^{+/+}$and $\mathrm{MerTK}^{-/-}$mice (Figure 4A). MerTK staining was detected in B16:F10 and MMTV$\mathrm{PyVmT}$ tumor macrophages grown in $\mathrm{MerTK}^{+++}$mice (Figure 4B). To verify that $\mathrm{MerTK}^{-/-}$macrophages were capable of infiltrating spontaneously forming tumors, we generated single cell suspensions from MMTV-PyVmT mice harboring $\mathrm{MerTK}^{-1-}$ and $\mathrm{MerTK}^{+/+}$ immune cells and stained cell suspensions for CD45, a cell surface marker of leukocytes, and F4/80. $\mathrm{MerTK}^{+/+}$and $\mathrm{MerTK}^{-/-} \mathrm{CD}^{4} 5^{+}$ leukocytes were detected at similar proportions of the total cell population (Figure 4C), revealing similar levels of $\mathrm{CD}^{4} 5^{+}$tumor leukocytes and comparable levels of $\mathrm{MerTK}^{+/+}$and $\mathrm{MerTK}^{-/-}$ tumor-associated macrophages in these established tumors.

Transcripts encoding the MerTK ligand, GAS6, were identified by qRT-PCR in RNA isolated from mammary glands 4 days after $M M T V-P y V m T$ tumor cell injection into the mammary fat pads of $\mathrm{MerTK}^{+/+}$and $\mathrm{MerTK}^{-/-}$mice. Gas6 levels in $\mathrm{MerTK}^{+/+}$mammary glands were $>3$-fold higher in tumor cell-injected glands as compared with those seen in the contralateral PBS-injected mammary gland, suggesting that GAS6 is elevated in the tumor microenvironment within 4 days (Figure 4D). Increased Gas6 mRNA levels were also detected in $\mathrm{MerTK}^{+/+}$samples harvested 4 days after B16:F10 cell inoculation, consistent with previous reports of elevated Gas6 levels in the tumor microenvironment (46). However, Gas6 remained unchanged in the $\mathrm{MerTK}^{-1-}$ mice after tumor cell inoculation with MMTV-PyVmT or B16:F10 cells. Because the $\mathrm{MerTK}^{-/-}$tumor microenvironment did not induce Gas 6 expression, it is possible that MerTK signaling may be part of the mechanism by which tumors upregulate GAS6 in the microenvironment. MFGE8 encodes another phosphatidylserine-interacting ligand used for efferocytosis by macrophages (47). Similar to Gas6, Mfge 8 transcript levels were elevated in response to tumors in $\mathrm{MerTK}^{+/+}$ but not $\mathrm{MerTK}^{-1-}$ samples (Figure 4D).

Cytokine expression in the microenvironment of established tumors was examined to determine whether the cytokine expres- sion patterns seen 4 days after tumor cell injection were sustained. Serum IL-12p70 was measured 3-4 weeks after mammary fat pad injection of MMTV-PyVmT tumor cells. As compared with that in tumor-bearing $\mathrm{MerTK}^{+/+}$mice, serum IL-12p70 was increased in tumor-bearing $\mathrm{MerTK}^{-/}$mice (Figure 4E), confirming that MerTK signaling in the tumor microenvironment suppresses proinflammatory cytokine production in both early and late stages of tumor progression. To specifically assess MerTK-dependent cytokine modulation in macrophages and DCs in the tumor microenvironment, we harvested CD $11 \mathrm{~b}^{+}$cells from transgenic MMTV-PyVmT mice 8 weeks after lethal irradiation and bone marrow transplantation from $\mathrm{MerTK}^{+/+}$or $\mathrm{MerTK}^{-/-}$donors. Protein lysates

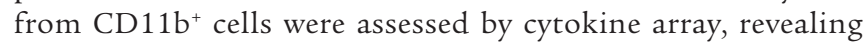
increased levels of IL-1 $\beta$, IL-6, and IL-12p40 in tumor-associated $\mathrm{MerTK}^{-/-} \mathrm{CD}_{11 \mathrm{~b}^{+}}$cells as compared with levels seen in $\mathrm{MerTK}^{+/+}$

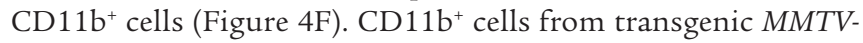
PyVmT mice harvested 15 weeks after bone marrow transplant revealed significantly elevated IL-6 levels in $\mathrm{MerTK}^{-/-}$tumor-associated CD11b cells (Figure 4G). M1- and M2-programmed tumor-associated $\mathrm{F} 4 / 80^{+}$macrophages were identified in situ using antibodies against inducible nitric oxide synthase (iNOS) and arginase-1 (Arg1), respectively. These results confirmed that the density of macrophages in the tumor microenvironment was relatively similar in tumors grown in $\mathrm{MerTK}^{+/+}$or $\mathrm{MerTK}^{-/-}$bone marrow recipients. Recipients of $\mathrm{MerTK}^{-/-}$bone marrow showed a modest decrease in the ratio of M2 to M1 tumor-associated macrophages as compared with what was seen in tumors harvested from $\mathrm{MerTK}^{+/+}$bone marrow recipients, although this trend was not statistically significant (Supplemental Figure 3). Taken together, these data demonstrate that targeted loss of MerTK from

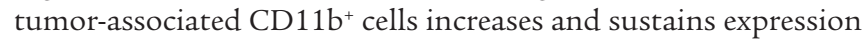
of proinflammatory cytokines, suggesting that MerTK signaling within tumor-associated monocyte-derived cells limits the expression of proinflammatory cytokines.

$\mathrm{CD}^{+}$T lymphocytes are more abundant in the $\mathrm{MerTK}^{-1-}$ tumor microenvironment. Four days after MMTV-PyVmT tumor cell inoculation into the mammary fat pad, mammary lymph nodes were removed and the remaining tissue was used to generate single cell suspensions. Staining for CD8 and CD4 revealed that the total $\mathrm{CD}^{+}{ }^{+} \mathrm{CD} 4-$ population was decreased in tumor-inoculated $\mathrm{MerTK}^{+/+}$samples (Figure 5A) but remained unchanged in tumor-inoculated $\mathrm{MerTK}^{-1-}$ mice. Because NK cells express MerTK and also express CD8, we measured the relative presence of NK cells in the early tumor microenvironment by staining the $\mathrm{CD} 8^{+} \mathrm{CD} 4^{-}$population with NK1.1. Although a slight increase in NK1.1 $1^{+} \mathrm{CD} 8^{+} \mathrm{CD} 4^{-}$levels was observed in tumor-inoculated $\mathrm{MerTK}^{+/+}$mice, this did not reach statistical significance (Figure 5B). Similar levels were seen in $\mathrm{MerTK}^{-/}$mice treated with either PBS or with tumor cells. While these results do not rule out the impact of MerTK on tumor-infiltrating NK cell behavior, these data suggest that NK cell numbers are not greatly affected in the early tumor microenvironment by MerTK. Immunohistochemical detection of granzyme B was used to detect cytotoxic leukocytes (such as CD8 ${ }^{+}$ T lymphocytes, NK cells, or NKT cells) in the tumor microenvironment of MMTV-PyVmT tumors 3-8 weeks after tumor transplant. Granzyme B-positive cells were more abundant in tumors grown in $\mathrm{MerTK}^{-1-}$ mice as compared with those in tumors grown in $\mathrm{MerTK}^{+/+}$littermates (Figure 5C). In contrast to what was seen with the $\mathrm{CD}^{+} \mathrm{CD} 4^{-}$population (Figure $5 \mathrm{~A}$ ), we found an early tumor-elicited increase in the $\mathrm{CD}^{-} \mathrm{CD}^{+}$population in $\mathrm{MerTK}^{++}$ 
A

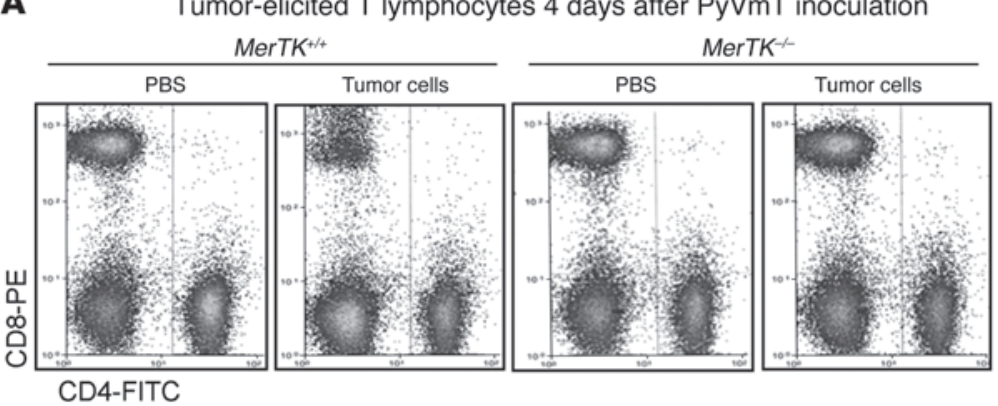

\section{B Tumor-elicited CD8+NK1.1+ cells}

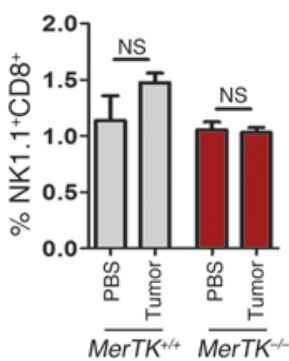

Tumor-elicited CD8 ${ }^{+} \mathrm{CD} 4$ - lymphocytes

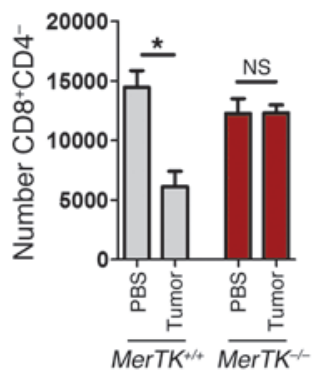

D Tumor-elicited CD8-CD4+ lymphocytes

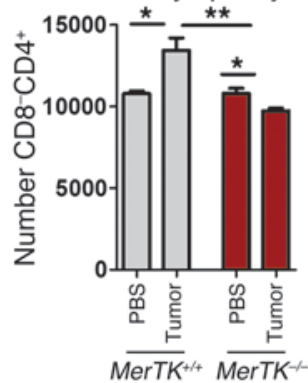

E

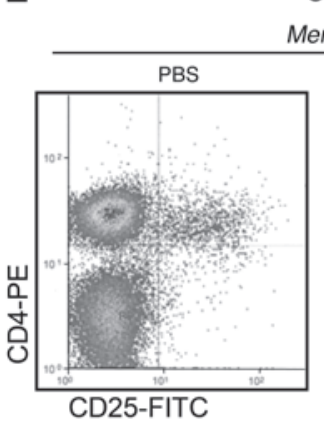

CD4+ Treg lymphocytes 4 days after inoculation

MerTK $^{+/ 4}$

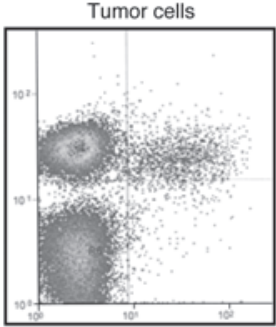

F Immunodepletion of $\mathrm{CD} 8^{+} \mathrm{T}$ lymphocytes

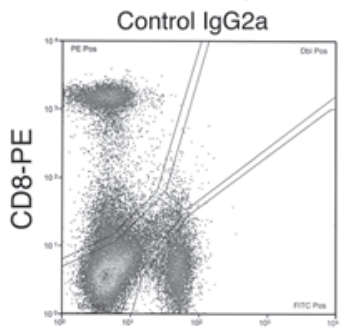

CD4-FITC

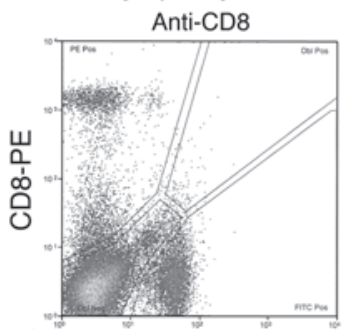

CD4-FITC

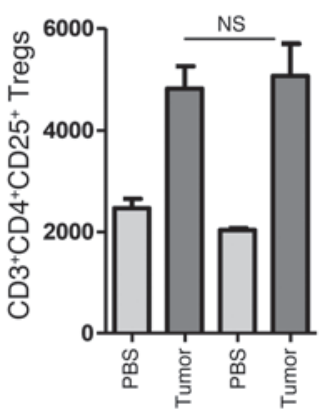

$\overline{\text { MerTK }^{+1+}} \overline{\text { MerTK }^{-1}}$
G PyVmT mammary tumor formation after $\mathrm{CD}^{+}$immunodepletion MerTK $^{-1-}$
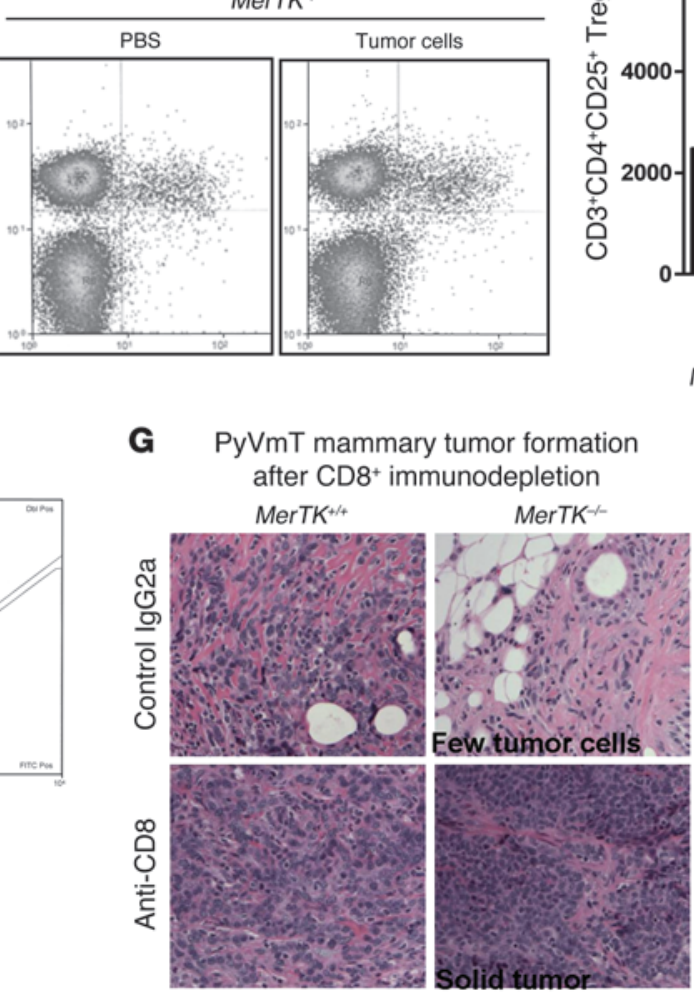

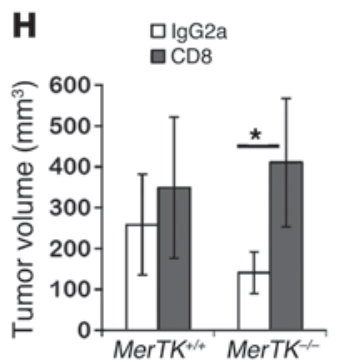




\section{Figure 5}

Increased presence of $\mathrm{CD}^{+} \mathrm{T}$ lymphocytes in $\mathrm{MerTK}^{-/-}$microenvironment is required for inhibition of tumor growth. (A and $\mathbf{B}$ ) Flow cytometric analysis of CD8/CD4- and NK1.1+CD8+-stained mammary gland suspensions 4 days after MMTV-PyVmT or PBS injection, with quantification $(n=6)$. (C) Immunohistochemistry for granzyme B. Original magnification, $\times 600$. (D and E) Flow cytometry and quantitation of (D) CD8-CD4+ and (E) CD25+CD4+ cells in mammary gland suspensions collected 4 days after intramammary injection of tumor cells or PBS. (F-H) MMTV-PyVmT tumor cells were injected into mammary fat pads of anti-CD8 antibody-treated mice. Mammary glands/ tumors were collected after 28 days for analysis. (F) CD8/CD4-stained tumor suspensions were assessed by flow cytometry. (G) Representative histological sections are shown. Original magnification, $\times 200 .(H)$ Average MMTV-PyVmT tumor volume at 28 days after inoculation is shown $\pm \operatorname{SD}(n=8)$. $P$ values were calculated using Student's $t$ test. ${ }^{\star} P<0.05 ;{ }^{* \star} P<0.01$.

mice but a slight decrease in $\mathrm{MerTK}^{-/-}$mice (Figure 5D). We further examined this population for expression of CD25 to identify Tregs. Similar basal levels of $\mathrm{CD} 4^{+} \mathrm{CD} 25^{+}$cells were identified 4 days after PBS injection into $\mathrm{MerTK}^{+/+}$and $\mathrm{MerTK}^{-/-}$mice (Figure $5 \mathrm{E})$. Although levels of $\mathrm{CD} 4^{+} \mathrm{CD} 25^{+}$cells increased in response to tumors, the increase was similar in $\mathrm{MerTK}^{+/+}$and $\mathrm{MerTK}^{-/-}$mice.

Depletion of $\mathrm{CD}^{+} \mathrm{T}$ lymphocytes restores tumor growth in $\mathrm{MerTK}^{-/}$ mice. $\mathrm{CD}^{+} \mathrm{T}$ lymphocytes were depleted from $\mathrm{MerTK}^{+/+}$and MerTK $K^{-1-}$ mice using antibodies against CD8. Mice were pretreated with anti-CD8 for 1 week prior to $M M T V-P y V m T$ tumor inoculation into mammary fat pads, and antibody treatment was maintained for 3 weeks following tumor inoculation. Tumor cell suspensions were stained with antibodies against CD4 and CD8, revealing decreased presence of $\mathrm{CD} 8{ }^{+} \mathrm{CD} 4^{-}$cells in the tumor microenvironment of mice treated with anti-CD8 antibody, as compared with those treated with isotype-matched antibody control (Figure 5F). Histological examination revealed that, while $\mathrm{MerTK}^{-1-}$ mice treated with the isotype control antibody developed small tumors comprising primarily matrix and stromal cells (Figure 5G), MerTK $^{-/}$mice treated with anti-CD8 antibodies developed larger, more aggressive tumors, with densely packed tumor epithelial cells, closely resembling the histological appearance of tumors grown in $\mathrm{MerTK}^{+/+}$mice (Figure 5G). Tumors grown in $\mathrm{MerTK}^{-1-}$ mice were nearly 3-fold larger upon CD8 depletion as compared with those grown in IgG2a-treated $\mathrm{MerTK}^{-/}$mice (Figure $5 \mathrm{H})$. These data suggest that, in the $\mathrm{MerTK}^{-1-}$ tumor microenvironment, $\mathrm{CD}^{+} \mathrm{T}$ lymphocytes are, at least in part, responsible for decreased tumor mass.

\section{Discussion}

The stromal microenvironment in which a tumor exists greatly influences its pathophysiology. Specifically, tumor-associated macrophages in human and mouse tumors have been correlated with poor prognosis and increased malignant progression. This was illustrated by studies in which targeted inhibition of CSF1 receptor signaling in tumor-associated macrophages, through gene targeting or through antibody-based pharmacologic strategies, decreased tumor growth and metastasis in mice $(37,38,41,48,49)$. Therefore, tumor macrophages are valid therapeutic targets. Macrophages and DCs can produce the cytokines and growth factors that encourage tumor progression and metastasis, such as IL-10, which dampen antitumor immune responses (40). We demonstrate here that the $\mathrm{MerTK}^{+/+}$microenvironment responds very rapidly after tumor cell implantation by increasing production of IL-10 and GAS6 and decreasing production of IL-12. These cytokine changes are correlated with a decreased local presence of $\mathrm{CD}^{+} \mathrm{T}$ lymphocytes in the tumor. IL-10 and GAS6 induction in the tumor microenvironment required MerTK, as tumor-induced IL-10 and GAS6 expression was not observed in MerTK-deficient mice. This is consistent with previous data suggesting that MerTK is required in the innate immune system to induce IL-10 following efferocytosis and TLR activation $(3,4,21,50,51)$. We also showed that MerTK-deficient cells in the tumor microenvironment express increased IL-12 and IL-6, proinflammatory cytokines known to be repressed by MerTK signaling in response to efferocytosis or TLR activation $(4,32)$.

Our results demonstrating upregulation of GAS6 in the tumor microenvironment of $\mathrm{MerTK}^{+/+}$mice are consistent with a previously published study, demonstrating GAS6 expression in tumor macrophages and showing that genetic loss of Gas 6 in the microenvironment of syngeneic tumor transplants decreases tumor growth (46). In these studies, GAS6 was not required for tumor infiltration by macrophages; this is consistent with our finding that tumor-associated macrophage numbers are not altered by loss of MerTK. MerTK was needed for full induction of GAS6 in the tumor microenvironment, supporting a model in which MerTK signaling induces GAS6 expression in tumor macrophages, creating positive feed-forward signaling through MerTK. Sustained MerTK signaling would maintain the repression of proinflammatory cytokines and induce wound healing/tolerogenic cytokines, ultimately resulting in decreased antitumor immunity. In the PyVmT models, we have demonstrated that the GAS6 receptors MerTK, AXL and TYRO3 are absent and, thus, the production or lack of production of GAS6 is irrelevant to the tumor cell per se.

Several studies using genetic disruption of MerTK, AXL, and TYRO3 in mice demonstrated that the innate immune system responds to pathogen-mimicking agents (TLR activation) with an early increase in IL-12, IL-6, and type I IFN production, followed by MerTK-, AXL-, and TYRO3-dependent reduction of proinflammatory cytokine expression, and finally with induction of wound healing and tolerogenic cytokines (IL-10, TGF- $\beta)(3,4,21,50,51)$. In the absence of these receptors, the innate immune system is unable to dampen expression of proinflammatory cytokines and unable to induce production of tolerogenic cytokines. Similarly, we found that early responses of the innate immune system in the $\mathrm{MerTK}^{+/+}$tumor microenvironment were characterized by dampened levels of IL-12 and increased levels of IL-10. However, loss of MerTK from the tumor microenvironment caused sustained IL-12 induction (for up to 14 weeks), without induction of IL-10. Therefore, it is possible that the innate immune system uses MerTK signaling in the tumor microenvironment in a way that parallels MerTK function in wounding responses.

Alternatively, MerTK in the tumor microenvironment may respond to efferocytosis. Apoptosis occurs in all tissues, and generally apoptosis rates are higher in rapidly proliferating tumors as compared with quiescent tissues. One major physiological role of MerTK is to clear apoptotic material through efferocytosis (23). To ensure that presentation of self-antigens does not stimulate autoimmunity following efferocytosis, macrophages suppress IL-12 production upon efferocytosis and induce IL-10 production (47). This limits $\mathrm{CD}^{+} \mathrm{T}$ lymphocyte expansion and reduces antigen presentation. It is possible that macrophage-mediated efferocytosis of apoptotic tumor cells drives the cytokine expression patterns that characterize tumor-associated macrophages. This scenario, 
while not proven, would predict that modulating the consequence of chronic efferocytosis, through targeted inhibition of MerTK, would increase IL-12 levels, decrease IL-10 levels, increase tumor $\mathrm{CD}^{+} \mathrm{T}$ lymphocytes, and decrease malignant progression. However, this hypothesis remains to be tested, requiring a systematic evaluation of efferocytosis in the tumor microenvironment. We did not observe major differences in apoptotic material in the microenvironment of $\mathrm{MerTK}^{+/+}$and $\mathrm{MerTK}^{-/-}$mice in late-stage tumors (Supplemental Figure 1). However, dramatic differences in apoptotic cell numbers are only seen in acute challenges in $\mathrm{MerTK}^{-1-}$ mice (23). Other macrophage receptors eventually clear apoptotic material, but these alternative clearance mechanisms do not suppress the inflammatory response to the extent that MerTK does. This conclusion is substantiated by the autoimmune phenotype seen in the $\mathrm{MerTK}^{-/}$mice.

IL-12 stimulates antitumor immunity in several models of epithelial tumors in part by promoting $\mathrm{CD}^{+} \mathrm{T}$ lymphocyte expansion (43). Because MerTK suppresses IL-12 production in the tumor microenvironment, MerTK signaling may suppress antitumor immunity through suppression of $\mathrm{CD}^{+} \mathrm{T}$ lymphocyte proliferation. Consistent with this idea, we found that depletion of $\mathrm{CD}^{+} \mathrm{T}$ lymphocytes restored tumor growth in the MerTK-deficient tumor microenvironment. In the case of AXL/TYRO3/MerTK triple-deficient mice, sustained IL-12 levels in normal tissues correlated with increased presence of $\mathrm{CD}^{+} \mathrm{T}$ lymphocytes and induction of adaptive immunity against self-antigens (21). While this response would be undesirable in normal tissues, immunity against tumor-specific antigens may improve the outcome of patients with cancer. This is supported by increasing evidence gained by expression microarrays that correlates tumors bearing a Th1 (e.g., IL-6, IL-12, CD8, granzyme B) gene expression signature with an improved outcome over tumors bearing a Th2 (e.g., IL-10, TGF- $\beta$, IL-4, cathepsin, IL-13, CD4) signature $(38,52,53)$. Recent work has shown that tumors can orchestrate the immune response in a manner advantageous for tumor growth, metastasis, and escape from immune surveillance (54). The present data suggest that inhibition of MerTK signaling may be a promising therapeutic intervention that can break the deleterious immune suppression cycle by directly targeting the tumor-associated innate immune system. This could shift the immune response toward a more favorable antitumor one. MerTK inhibition that would set the stage for an antitumor Th1 response combined with new clinical agents designed to prolong antitumor $\mathrm{T}$ cell action may well provide an additive antitumor effect.

\section{Methods}

Mice. All mice regardless of genotype were inbred to C57BL/ 6 for at least 10 generations. $\mathrm{MerTK}^{-/-}$mice, originally referred to as $\mathrm{Mer}^{\mathrm{KD}}$ mice, have been previously described (30).To generate $\mathrm{MerTK}^{+/+}$and $\mathrm{MerTK}^{-/-}$siblings, $\mathrm{MerTK}^{+/-}$males and females were mated to generate the expected Mendelian ratios of offspring, which were genotyped using the following primers: forward 5'-GAATTTACCTTTCACAGGTTGCGG; reverse 5'-TCGTCAAGAAGGCGATAGAAGGCG. Mice were maintained in American Association for Accreditation of Laboratory Animal Care-approved animal facilities at the University of North Carolina under an approved IACUC protocol. Where indicated, mice were treated with $\mathrm{BrdU}(10 \mathrm{mg} / \mathrm{kg}$ in sterile PBS) by i.p. injection 1 hour prior to tissue collection.

Transplantation of tumor cells. Primary mammary tumor cells were derived from a female MMTV-PyVmT mouse inbred to C57BL/ 6 for 6 generations as previously described (54). B16:F10 cells were obtained from American Type Tissue Culture Collection. For orthotopic injections into the mam- mary fat pads, $10^{6} M M T V-P y V m T$ cells were resuspended in $100 \mu \mathrm{l}$ sterile PBS (in the presence or absence of $0.5 \times 10^{6}$ mouse mammary fibroblasts harvested from 6-week-old virgin $\mathrm{MerTK}^{+/+}$or $\mathrm{MerTK}^{-/-}$female C57BL/6 mice) and injected centrally in the right no. 4 inguinal fat pad of 6-weekold virgin mice. For orthotopic injections into the dermis, 1033 B16:F10 melanoma cells were resuspended in $100 \mu \mathrm{l}$ PBS and injected intradermally between the scapulae of 6 -week-old mice. For subcutaneous injection, $1 \times 10^{4} \mathrm{MC} 38$ colon cancer cells were injected into the flank. Tumors were detected by manual palpation. Statistical analysis of average tumor latency was assessed by log-rank test. Where indicated, i.p. injection of $3 \times 10^{3} \mathrm{~B} 16: \mathrm{F} 10$ cells was performed in $100 \mu \mathrm{l} \mathrm{PBS}$, and cells were collected in MEM by peritoneal lavage after 4 days.

Histological analysis and immunobistochemistry. Tumors and mammary glands were harvested and immediately fixed in 10\% formalin (VWR Scientific). Paraffin-embedded tumors and mammary glands were sectioned $(5 \mu \mathrm{m})$, rehydrated, and peroxidase-quenched with $3 \% \mathrm{H}_{2} \mathrm{O}_{2}$. Rehydrated slides were stained with H\&E by the UNC Lineberger Animal Histopathology Core Facility. Immunohistochemistry was performed as previously described $(55,56)$ using the following polyclonal antibodies: F4/80 (6A545, Santa Cruz Biotechnology Inc.; diluted 1:200), MerTK (produced by the Earp laboratory) against a peptide in the intracellular domain of human MerTK; diluted 1:100), pan-cytokeratin (H240, Santa Cruz Biotechnology Inc.; diluted 1:500), and granzyme B (Santa Cruz Biotechnology Inc.; diluted 1:200). Slides were washed in PBST and then incubated in biotinylated anti-rabbit or anti-rat antibody (Vector Laboratories) and developed using the Vectastain Kit (Vector Laboratories). Sections were photographed using the Zeiss LCM 210 microscope and Scion Image 2.0 software.

Western blot analysis. MMTV-PyVmT tumor cells were lysed in lysis buffer (50 mM HEPES, pH 7.5, $150 \mathrm{mM} \mathrm{NaCl}, 10 \mathrm{mM}$ EDTA, 10\% glycerol, and $1 \%$ Triton $\mathrm{X}-100)$ supplemented with phosphatase inhibitors $(1 \mathrm{mM}$ $\mathrm{Na}_{3} \mathrm{VO}_{4}$ and $0.1 \mathrm{mM} \mathrm{Na}_{2} \mathrm{MoO}_{4}$ ) and protease inhibitors (Complete Mini, Roche Applied Science). Whole spleen lysates harvested from $\mathrm{MerTK}^{+/+}$and $\mathrm{MerTK}^{-/-}$mice and whole brain lysates harvested from $\mathrm{Ty}_{\mathrm{ro}} 3^{+/+}$and Tyro3 $3^{-/-}$ mice were prepared in sodium chloride-Tris-EDTA buffer and sonicated. Whole protein lysates were separated on $8 \%$ Tris-glycine SDS-PAGE gels (Life Technologies) and then transferred to nitrocellulose membranes (iBlot, Life Technologies). Membranes were probed with primary antibodies: anti-mouse MerTK (AF591, R\&D Systems; diluted 1:2,000), anti-mouse AXL (AF854, R\&D Systems; diluted 1:1,000), and anti-TYRO3 rabbit sera (produced by the Prieto laboratory; diluted 1:20,000)

Bone marrow transplantation. Six-week-old female MMTV-PyVmT mice were lethally irradiated with 9.5 Gy split over 2 doses. $1 \times 10^{6}$ bone marrow cells from C57BL/6-Tg(UBC-GFP) 30Scha/J (The Jackson Laboratory) MerTK ${ }^{+/+}$ or $\mathrm{MerTK}^{-/}$donors were delivered to irradiated mice by tail vein injection. After 4 weeks, recipient mice that expressed $\geq 75 \%$ GFP in peripheral blood indicated successful engraftment and were included for further analysis. Animals were housed and experimental procedures were done in accordance with the regulatory standards approved by the University of Colorado, Anschutz Medical Campus' IACUC.

Tumor measurement by MRI. Mammary gland tumor growth was measured over time by MRI. Mice were anesthetized with $1.5 \%$ to $2.5 \%$ inhaled isoflurane and were placed in the 4.7 Tesla Bruker MRI/MRS PharmaScan. Gadolinium-DTPA bismethylamide (gadodiamide, OMNISCAN) was administered intravenously as a contrast agent. Axial plane images of the animals were obtained using conventional T1-, T2-, and proton densityweighted MRI. Additional fast imaging with steady-state precession (FISP) images were required in order to exclude cysts from the tumor volume calculations, and all images were processed using Bruker ParaVision software. The total tumor volume of each tumor was calculated from the resulting images by multiplying the pixel volume by the number of pixels within 
the tumor area by hand-tracing the ROI with "track" command from each set of slices. All MRI acquisitions and data analysis were performed at the University of Colorado Cancer Center Animal Imaging Core.

qRT-PCR. Total RNA was isolated using RNeasy (Qiagen) from single mammary cell suspensions and from peritoneal lavage collections. Total RNA (10 ng) was reverse transcribed with transcript-specific primers and then amplified with transcript-specific primers using SyBR Green (Invitrogen), according to manufacturer's instructions, with a temperature of $62^{\circ} \mathrm{C}$. The following primers and probes were used. Gas6, $5^{\prime}$-TCTTCTCACACTGTGCTGTTGCG and 5'-GGTCAGGCAAGTTCTGAACACAT; mouse Il12p40, 5'-TTATGTTGTAGAGGTGGACTGG and 5'-TTTCTTTGCACCAGCCATGAGC; mouse $I l 10,5^{\prime}$-TGGCCCAGAAATCAAGGAGC and 5'-CAGCAGACTCAATACACACT; mouse $M f g e 8$, forward: 5'-GGGCCTGAAGAATAACACGA and 5'-AGGGCAACTTGGACAACAAC; and mouse Gapdh, 5'-AACGACCCCTTCATTGAC-3' and 5'-TCCACGACATACTCAGCAC. The CT for each transcript within each sample was corrected for the CT of Gapdh within each sample, normalized to the CT of a single sample, and converted into relative levels of expression using the $\delta \delta \mathrm{CT}$ method, such that all values are presented as a fold change in reference to a single sample. Samples were analyzed 6 times.

ELISA. Serum was collected by cardiac puncture and immediately stored in frozen aliquots. Neat serum was analyzed by ELISA for mouse IL-12p70 (R\&D Systems) and IL-6 (R\&D Systems) according to manufacturer's directions. Standard curves generated using manufacturer-supplied IL-12 and IL- 6 were used to calculate serum concentrations of each protein.

Flow cytometry. Single cell suspensions of tumors or mammary glands were generated by mechanical tissue disruption using razor blades followed by 30 minutes in $1 \mathrm{mg} / \mathrm{ml}$ collagenase A (Sigma-Aldrich) in DMEM, 10 minutes in $1 \mathrm{mg} / \mathrm{ml}$ dispase (BD Biosciences), 2 minutes in $0.25 \%$ trypsin (BD Biosciences), and 5 minutes in DNase I (Stem Cell Technologies). Single cell splenocyte suspensions were generated by forcing spleens through $70-\mu$ and $40-\mu$ mesh filters. Cells $\left(1.5 \times 10^{6}\right)$ were blocked with mouse Fc $\gamma$ III/II Receptor Block (BD Biosciences) for 10 minutes prior to 30-minute incubation at room temperature with the following fluorescence-conjugated BD Pharmingen antibodies (BD Biosciences) diluted 1:200: rat antimouse Thy1 (53-2.1), rat anti-mouse CD8 (53-6.7), rat anti-mouse CD4 (GK1.5), rat anti-mouse CD3 (500A2), rat anti-mouse CD45 (30F11), rat anti-mouse CD11b (M1/70), hamster anti-mouse CD11c (HL3), and mouse IgG2a anti-mouse NK1.1 (PK136). Alternatively, splenocytes were fixed and permeabilized (BD Cytofix/Cytoperm, BD Biosciences), DNA was denatured using $1 \mathrm{M} \mathrm{HCl}$ for 15 minutes, and cells were stained with FITC-conjugated anti-BrdU antibody (1:100, BD Biosciences) for 1 hour. Cells were washed and counterstained with propidium iodide. Cells were analyzed at the UNC Flow Cytometry Core Facility on a Cytek-modified 5-color FACSCalibur using hardware single-color compensation.

Tumor-associated macrophage isolation. CD11 $\mathrm{b}^{+}$tumor-associated macrophages were isolated from freshly digested (serum-free DMEM with $0.1 \%$ Collagenase I [Sigma-Aldrich] and DNaseI [EMD Millipore] at $37^{\circ} \mathrm{C}$ for 45 minutes) single cell suspensions, which were enriched using CD11b magnetic beads (Miltenyi Biotec) and MS Columns (Miltenyi Biotec) according to the manufacturer's directions.
Cytokine array. Whole cell lysates were prepared in lysis buffer $(50 \mathrm{mM}$ HEPES, pH 7.5, $150 \mathrm{mM} \mathrm{NaCl}, 10 \mathrm{mM}$ EDTA, 10\% glycerol, and 1\% Triton X-100) supplemented with phosphatase inhibitors (1 $\mathrm{mM} \mathrm{Na}_{3} \mathrm{VO}_{4}$ and $0.1 \mathrm{mM} \mathrm{Na}_{2} \mathrm{MoO}_{4}$ ) and protease inhibitors (Complete Mini, Roche Applied Science). Relative protein expression was analyzed on custom cytokine arrays, using $100 \mu \mathrm{g}$ lysates according to the manufacturer's instructions (RayBiotech). The cytokine membranes were quantified by densitometric analysis using Image $1.44 \mathrm{o}$ (NIH) with the MicroArray Profile plug-in (OptiNav).

Antibody-mediated immune-depletion of $C D 8^{+}$cells. Mice were treated by i.p. injection of anti-mouse CD8 antibody (clone 53.6.7, BioXCell) at $2.5 \mathrm{mg} / \mathrm{kg}$ in sterile PBS twice weekly for 2 weeks prior to inoculation with MMTVPyVmT tumor cells and afterward for an additional 4 weeks.

In situ identification of TUNEL-positive cells and macrophage-programming markers. Immunofluorescent in situ staining of apoptotic cells in paraffin-embedded tumors and mammary glands was done using the Dead End Fluorometric TUNEL system (Promega). Sections were subsequently stained with anti-F4/80-PE (Serotec; diluted 1:100) and DAPI. Triple staining to identify macrophage-programming status was performed using antibodies against F4/80-PE (Serotec; diluted 1:100), iNOS-FITC (BD Biosciences; diluted 1:100), and Arginase I-AMCA (Santa Cruz Biotechnology Inc.; 1:100) as previously described (57). Images were acquired on a Zeiss Axioplan 2 epifluorescent microscope.

Statistics. Data represent the mean \pm SEM of all experiments, unless otherwise noted. Statistical significance was calculated by Mantel-Cox test (tumor latency), Student's $t$ test, Student's $t$ test with Welch's correction for unequal variance (tumor weight, tumor volume), or analysis of variance using Prism 5 (GraphPad Software) unless otherwise noted.

Study approval. All studies involving mice were performed with approval from the IACUCs of the University of North Carolina, Chapel Hill, North Carolina, USA, or the University of Colorado, Anschutz Medical Campus.

\section{Acknowledgments}

This work was supported by the Breast Cancer Research Foundation (to H.S. Earp III), the UNC Breast Cancer SPORE P50 CA058223 (to H.S. Earp III), NIH R01 CA143126 (to R.S. Cook), VICC Breast Cancer SPORE P50 CA98131, and Susan G. Komen for the Cure KG100677 (to R.S. Cook). The Animal Imaging Core at University of Colorado is supported by the following grants: NCI Cancer Center P30 CA046934; NCRR CTSA UL1 RR025780. The authors thank Kendra Huber (RT MRI/CT) for her help with all MRI studies and data analysis.

Received for publication November 8, 2012, and accepted in revised form May 10, 2013.

Address correspondence to: H. Shelton Earp III, UNC Lineberger Comprehensive Cancer Center, University of North Carolina Chapel Hill, 450 West Avenue, CB 7295, $1^{\text {st }}$ Floor Administration Office, Chapel Hill, North Carolina 27599, USA. Phone: 919.966.2335; Fax: 919.966.3015; E-mail: hse@med.unc.edu.

\footnotetext{
1. Linger RM, Keating AK, Earp HS, Graham DK. Taking aim at Mer and Axl receptor tyrosine kinases as novel therapeutic targets in solid tumors. Expert Opin Ther Targets. 2010;14(10):1073-1090.

2. Linger RM, Keating AK, Earp HS, Graham DK. TAM receptor tyrosine kinases: biologic functions, signaling, and potential therapeutic targeting in human cancer. Adv Cancer Res. 2008;100:35-83.

3. Lemke G, Rothlin CV. Immunobiology of the TAM receptors. Nat Rev Immunol. 2008;8(5):327-336.
}

4. Rothlin CV, Lemke G. TAM receptor signaling and autoimmune disease. Curr Opin Immunol. 2010; 22(6):740-746.

5. Brown J, Krodel M, Pazos M, Lai C, Prieto A. Crossphosphorylation, signaling and proliferative functions of the Tyro- 3 and Axl receptors in Rat 2 cells. PLoS One. 2012;7(5):e36800.

6. Graham DK, Dawson TL, Mullaney DL, Snodgrass HR, Earp HS. Cloning and mRNA expression analysis of a novel human protooncogene, c-mer. Cell
Growth Differ. 1994;5(6):647-657.

7. Nagata K, et al. Identification of the product of growth arrest-specific gene 6 as a common ligand for Axl, Sky, and Mer receptor tyrosine kinases. J Biol Chem. 1996;271(47):30022-30027.

8. Varnum B, et al. Axl receptor tyrosine kinase stimulated by the vitamin K-dependent protein encoded by growth-arrest-specific gene 6. Nature. 1995;373(6515):623-626.

9. Hafizi S, Dahlback B. Gas6 and protein S. Vitamin K- 
dependent ligands for the Axl receptor tyrosine kinase subfamily. FEBS J. 2006;273(23):5231-5244.

10. Stitt TN, et al. The anticoagulation factor protein S and its relative, Gas6, are ligands for the Tyro 3 Axl family of receptor tyrosine kinases. Cell. 1995; 80(4):661-670

11. Caberoy NB, Zhou Y, Li W. Tubby and tubby-like protein 1 are new MerTK ligands for phagocytosis. EMBO J. 2010;29(23):3898-3910.

12. Caberoy NB, Alvarado G, Bigcas J, Li W. Galectin-3 is a new MerTK-specific eat-me signal. J Cell Physiology. 2011;227(2):401-407.

13. Wang Y, et al. Mer receptor tyrosine kinase promotes invasion and survival in glioblastoma multiforme. Oncogene. 2013;32(7):872-882.

14. Png KJ, Halberg N, Yoshida M, Tavazoie SF. A microRNA regulon that mediates endothelial recruitment and metastasis by cancer cells. Nature. 2012 481(7380):190-194.

15. Jansen FH, et al. Profiling of antibody production against xenograft-released proteins by protein microarrays discovers prostate cancer markers. J Proteome Res. 2012;11(2):728-735.

16. Tworkoski K, et al. Phosphoproteomic screen identifies potential therapeutic targets in melanoma. Mol Cancer Res. 2011;9(6):801-812.

17. Keating AK, et al. Inhibition of Mer and Axl receptor tyrosine kinases in astrocytoma cells leads to increased apoptosis and improved chemosensitivity. Mol Cancer Ther. 2010;9(5):1298-1307.

18. Graham DK, et al. Ectopic expression of the protooncogene Mer in pediatric T-cell acute lymphoblastic leukemia. Clin Cancer Res. 2006;12(9):2662-2669.

19. Keating AK, et al. Lymphoblastic leukemia/lymphoma in mice overexpressing the Mer (MerTK) receptor tyrosine kinase. Oncogene. 2006;25(45):6092-6100

20. Behrens EM, Gadue P, Gong SY, Garrett S, Stein PL, Cohen PL. The mer receptor tyrosine kinase: expression and function suggest a role in innate immunity. Eur J Immunol. 2003;33(8):2160-2167.

21. Rothlin CV, Ghosh S, Zuniga EI, Oldstone MB Lemke G. TAM receptors are pleiotropic inhibitors of the innate immune response. Cell. 2007; 131(6):1124-1136

22. Fadok VA, Voelker DR, Campbell PA, Cohen JJ, Bratton DL, Henson PM. Exposure of phosphatidylserine on the surface of apoptotic lymphocytes triggers specific recognition and removal by macrophages. J Immunol. 1992;148(7):2207-2216.

23. Scott RS, et al. Phagocytosis and clearance of apoptotic cells is mediated by MER. Nature. 2001; 411(6834):207-211.

24. Seitz HM, Camenisch TD, Lemke G, Earp HS, Matsushima GK. Macrophages and dendritic cells use different Axl/Mertk/Tyro3 receptors in clearance of apoptotic cells. J Immunol. 2007;178(9):5635-5642.

25. Tibrewal N, et al. Autophosphorylation docking site Tyr-867 in Mer receptor tyrosine kinase allows for dissociation of multiple signaling pathways for phagocytosis of apoptotic cells and down-modulation of lipopolysaccharide-inducible NF-kappaB transcriptional activation. J Biol Chem. 2008;283(6):3618-3627.

26. Filardy AA, et al. Proinflammatory clearance of apoptotic neutrophils induces an IL-12(low) IL-10(high) regulatory phenotype in macrophages. J Immunol. 2010;185(4):2044-2050.

27. Gal A, et al. Mutations in MERTK, the human orthologue of the RCS rat retinal dystrophy gene, cause retinitis pigmentosa. Nat Genet. 2000;26(3):270-271.

28. Sandahl M, Hunter DM, Strunk KE, Earp HS, Cook RS. Epithelial cell-directed efferocytosis in the post-partum mammary gland is necessary for tissue homeostasis and future lactation. BMC Dev Biol. 2010;10:122.

29. Cohen PL, et al. Delayed apoptotic cell clearance and lupus-like autoimmunity in mice lacking the c-mer membrane tyrosine kinase. J Exp Med. 2002; 196(1):135-140.

30. Camenisch TD, Koller BH, Earp HS, Matsushima GK. A novel receptor tyrosine kinase, Mer, inhibits TNFalpha production and lipopolysaccharide-induced endotoxic shock. J Immunol. 1999;162(6):3498-3503.

31. Shao WH, Eisenberg RA, Cohen PL. The Mer receptor tyrosine kinase is required for the loss of $\mathrm{B}$ cell tolerance in the chronic graft-versus-host disease model of systemic lupus erythematosus. J Immunol. 2008;180(11):7728-7735

32. Shao WH, et al. Disrupted Mer receptor tyrosine kinase expression leads to enhanced MZ B-cell responses. J Autoimmun. 2010;35(4):368-374.

33. Lu Q, et al. Tyro-3 family receptors are essential regulators of mammalian spermatogenesis. Nature. 1999;398(6729):723-728.

34. Pollard JW. Tumour-educated macrophages promote tumour progression and metastasis. Nat Rev Cancer. 2004;4(1):71-78

35. Pollard JW. Trophic macrophages in development and disease. Nat Rev Immunol. 2009;9(4):259-270.

36. Ruffell B, Affara NI, Coussens LM. Differential macrophage programming in the tumor microenvironment. Trends Immunol. 2012;33(3):119-126.

37. Lin EY, Nguyen AV, Russell RG, Pollard JW. Colony-stimulating factor 1 promotes progression of mammary tumors to malignancy. J Exp Med. 2001; 193(6):727-740.

38. Denardo DG, et al. Leukocyte complexity predicts breast cancer survival and functionally regulates response to chemotherapy. Cancer Discov. 2011; 1(1):54-67.

39. Wyckoff J, et al. A paracrine loop between tumor cells and macrophages is required for tumor cell migration in mammary tumors. Cancer Res. 2004 64(19):7022-7029.

40. Qian B, et al. A distinct macrophage population mediates metastatic breast cancer cell extravasation, establishment and growth. PLoS One. 2009;4(8):e6562.

41. Qian BZ, Pollard JW. Macrophage diversity enhances tumor progression and metastasis. Cell. 2010; 141(1):39-51

42. Grivennikov SI, Greten FR, Karin M. Immunity, inflammation, and cancer. Cell. 2010;140(6):883-899.

43. Trinchieri G. Interleukin-12 and the regulation of innate resistance and adaptive immunity. Nat Rev Immunol. 2003;3(2):133-146.

44. Guy CT, Cardiff RD, Muller WJ. Induction of mammary tumors by expression of polyomavirus middle $T$ oncogene: a transgenic mouse model for metastatic disease. Mol Cell Biol. 1992;12(3):954-961.

45. Kidd S, et al. Origins of the tumor microenvironment: quantitative assessment of adipose-derived and bone marrow-derived stroma. PLoS One. 2012; 7(2):e30563.

46. Loges S, et al. Malignant cells fuel tumor growth by educating infiltrating leukocytes to produce the mitogen Gas6. Blood. 2010;115(11):2264-2273.

47. Friggeri A, Yang Y, Banerjee S, Park YJ, Liu G, Abraham E. HMGB1 inhibits macrophage activity in efferocytosis through binding to the alphavbeta3-integrin. Am J Physiol Cell Physiol. 2010; 299(6):C1267-C1276.

48. Nowicki A, et al. Impaired tumor growth in colony-stimulating factor 1 (CSF-1)-deficient, macrophage-deficient op/op mouse: evidence for a role of CSF-1-dependent macrophages in formation of tumor stroma. Int J Cancer. 1996;65(1):112-119.

49. O'Brien J, et al. Alternatively activated macrophages and collagen remodeling characterize the postpartum involuting mammary gland across species. Am J Pathol. 2010;176(3):1241-1255.

50. Lemke G, Lu Q. Macrophage regulation by Tyro 3 family receptors. Curr Opin Immunol. 2003; 15(1):31-36.

51. Lu Q, Lemke G. Homeostatic regulation of the immune system by receptor tyrosine kinases of the Tyro 3 family. Science. 2001;293(5528):306-311.

52 . Kristensen $\mathrm{VN}$, et al. Integrated molecular profiles of invasive breast tumors and ductal carcinoma in situ (DCIS) reveal differential vascular and interleukin signaling. Proc Natl Acad Sci U S A. 2012; 109(8):2802-2807.

53. Teschendorff AE, et al. Improved prognostic classification of breast cancer defined by antagonistic activation patterns of immune response pathway modules. BMC Cancer. 2010;10:604.

54. Coussens L, Zitvogel L, Palucka K. Neutralizing tumor-promoting chronic inflammation: a magic bullet? Science. 2013;339(6117):286-291.

55. Cook RS, et al. ErbB3 ablation impairs PI3K/ Akt-dependent mammary tumorigenesis. Cancer Res. 2011;71(11):3941-3951.

56. Balko JM, et al. The receptor tyrosine kinase ErbB3 maintains the balance between luminal and basal breast epithelium. Proc Natl Acad Sci U S A. 2012;109(1):221-226

57. Redente EF, Orlicky DJ, Bouchard RJ, Malkinson AM. Tumor signaling to the bone marrow changes the phenotype of monocytes and pulmonary macrophages during urethane-induced primary lung tumorigenesis in A/J mice. Am J Pathol. 2007; 170(2):693-708 